\title{
Multichannel Spectroellipsometric Technology for Aguatic Environment Diagnostic
}

\author{
V.F. Krapivin, F.A. Mkrtchyan* \\ Institute of Radio Engineering and Electronics RAS, Fryazino, 141190 Moscow Regions, Russia \\ *Corresponding Author: ferd47@mail.ru
}

Copyright (C) 2014 Horizon Research Publishing All rights reserved.

\begin{abstract}
A compact measuring - information multi-channel spectroellipsometric system for monitoring the quality of aquatic environment, that is based on the combined use of spectroellipsometry and training, classification, and identification algorithms is described. This system is differed from modern analogues by the use of a new and very promising method of ellipsometric measurements, an original element base of polarization optics and a complex mathematical approach to estimating the quality of a water object subjected to anthropogenic influence.The spectroellipsometric system can be used in different fields where the water quality should be estimated or the presence of a particular set of chemical elements should be revealed. The system solves these problems by real-time monitoring of the aquatic environment. In the stationary version it allows the tracking of the dynamics of water quality in a stream, and when placed on a ship, it allows the measurement of water parameters along the route.Some experimental data and calculations are given. Assessment of the system precision is realized for water reservoirs located in South Vietnam.
\end{abstract}

Keywords Water Pollution, Spectroellipsometer, Algorithm, Expert System, Identification, Spectrum

\section{Introduction}

Problem of the aquatic environment operative diagnostics is arisen practically in many human base including agriculture, medicine, industry and service. The development of these anthropogenic processes poses the problem of water pollution control and synthesizing monitoring system for water quality assessment in active regime.

Pollutant sources can have different structures and classes. Therefore, monitoring system is to be multi-purpose and mobile. It can be realized with the use of spectroellipsometric technology that gives possibility to have the spectrums with the sensitivity of $0.01 \%[1,5,4,7]$.
The creation of multi-channel polarization optical instrumentation and use of spectroellipsometric technology are very important for the real-time ecological control of aquatic environment. It should be mentioned that efficient solution of this multi-parametric problem greatly depends on the precision and simplicity of ellipsometric devices. A technology of combined use of spectroellipsometry and algorithms of optical spectrums identification and recognition allows the creation of a standard integral complex of instrumental, algorithmic, modular and software tools for the collection and processing of data on the aquatic environment quality with forecasting and decision - making functions $[2,3]$.

Some realizations of the spectroellipsometric technology were used for water quality control in South Vietnam (Cao Van Phuong et al., 2009; Nguyen Xuan Man et al., 2011). Pollutant sources in Vietnam were grouped in classes: agriculture sewage; domestic, municipal, and industrial waste; shipping; aquaculture; and coastal mining. The expert system for ecological control was developed as part of an agreement between the Russian Academy of Sciences and the Vietnamese Academy of Sciences and Technology to collaborate on ecoinformatics and remote sensing. Specific attention was given for the water quality control in the estuary zones taking into consideration of pollution sources compounded in territories bordering the South China Sea.

This paper describes new version of spectroellipsometric system that develops and realizes the functions of contaminant identification for multi-component solutions. It is realized by means of combined use of cluster analysis and algorithms for inverse task solution.

In this paper, we propose a new method ellipsometric measurements - ellipsometry with a binary modulation of the polarization state ( BMPS ) in which the sample is to alternately directed radiation with two polarization states and analyzes the signal at the photodetector. In developed ellipsometry devices BMPS with no moving polarization elements combined with high sensitivity, stability and measurement speed. This approach to solving the problem in the foreign studies spectroellipsometry not known. 


\section{Spectroellipsometric Expert System for the Aquatic Environment Control}

Spectroellipsometric expert system for the aquatic environment diagnostics (SESAED) consists of measuring subsystem (Fig. 1) and software. The system's structure includes a compact multi-channel spectroellipsometer (MCS), information interface with computer (IIC), software (STW), and extending database (EDB). The STW realizes a number of algorithms to process data fluxes from the MCS and provides the service functions of visualization and control of measurements. The EDB consists of sets of standard spectral images of aquatic environments represented by points in the multi-dimensional vector space of indicators, pre-calculated on the basis of learning samples.

The principle behind the SESAED is based on fixing changes in light flux the MCS and digitizing them in IIC. Further processing of these data to make them more efficient is determined by STW with algorithms capable of recognizing spectral images. The degree of adaptability of the recognition procedure is determined by the level of accumulated knowledge about intensity fluctuations and the polarizing properties of light reflected from the water surface or dispersed and refracted within the water environment. The STW enables, in case of uncertain identification of spectral images, to make an expert decision based on the visual analysis of a spectral image. This procedure is realized in dialog mode with the SESAED and, if a decision is made, the operator can fix it in the database in the form of a standard for subsequent appearances of similar spectral images.

The principal structure of the STW unit providing the identification procedyre involves series algorithms (Table 1). The light intensity ${ }^{\zeta}{ }_{j}$ recorded at time $t_{i}$ in the channel $\lambda_{j}$ forms two spectra that correspond to different light polarizations. Operator takes decision what algorithm will be used for identification task solution.

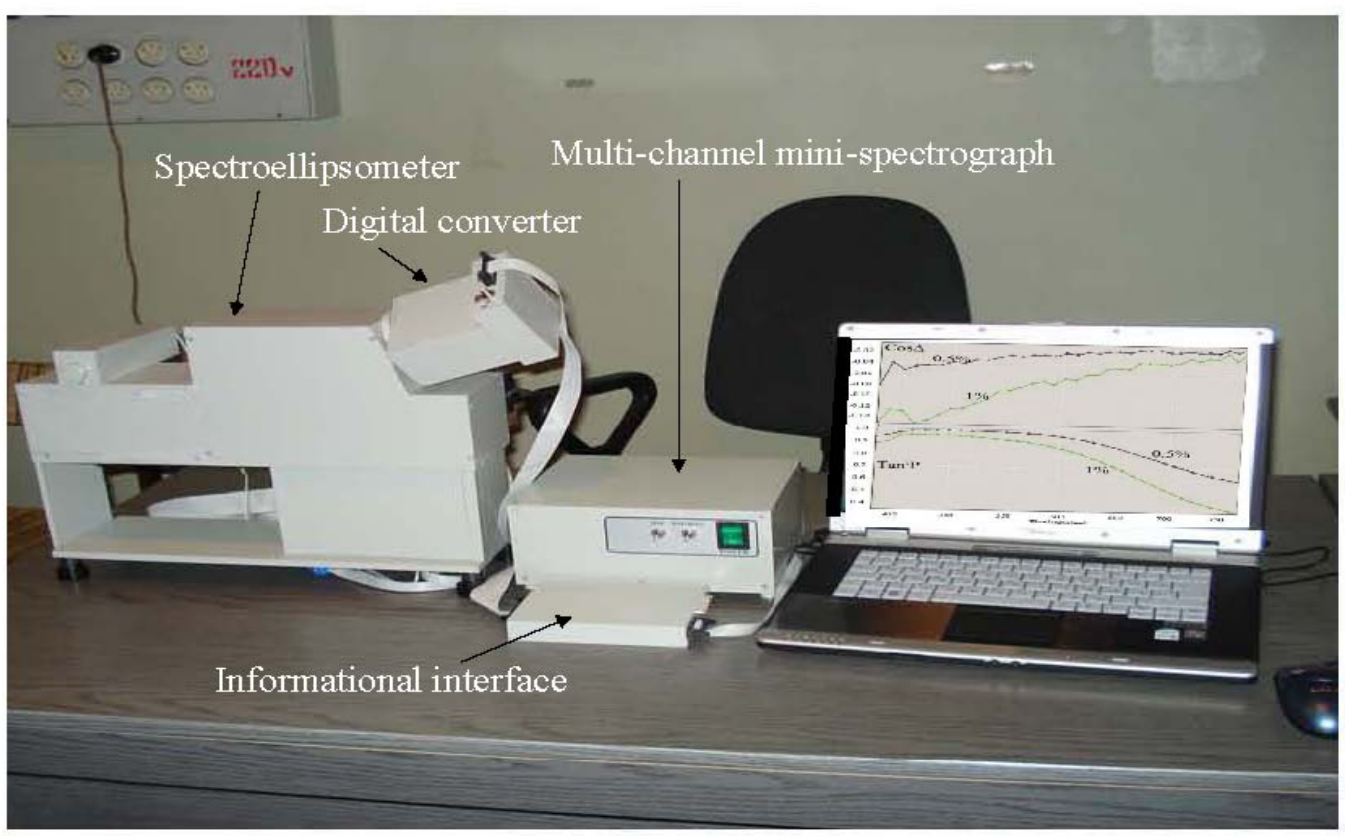

Figure 1. General view of the spectroellipsometric system.

Table 1. Description of the SESAED units

\begin{tabular}{|c|c|}
\hline Unit & Characteristic of the unit \\
\hline VOD & Visualization of observed data (spectra, correlations, statistical parameters, empirical and theoretical functions of distribution) \\
\hline CPCP & Calculation of parameters for the classical procedure of decision making when using the Neuman-Pearson criterion \\
\hline CSP & Calculation of statistical parameters and other characteristics, formation of the vector space $\left\{\mathrm{u}_{\mathrm{i}}\right\}$ \\
\hline EPPW & Estimation of parameters for the Wald sequential analysis in decision making \\
\hline REDF & Reconstruction of empirical distribution functions \\
\hline RFUI & Reconstruction of the functions of a user's interface equipped with software to intervene in the working regime of an expert system \\
\hline as any stage
\end{tabular}


After the learning procedure, the expert system is limited only by the volume of measurements fixed by the operator and proceeds from statistical reliability and the real-time regime. The operator has two possibilities to regulate this regime: establishing the volume of the series $\left\{\xi_{j}^{i}\right\}$ or fixing the time of their accumulation.

Usually the latter characteristic is chosen equal to one second. The operator is combined with SESAED units by means of the man/machine interface IIC, which provides selective control of operations in all units.

In the event of an oil slick on the water surface the system analyzes its thickness, age, source and geometry. The most informative measurements here are those made at $398 \mathrm{~nm}$, $439 \mathrm{~nm}$ and $480 \mathrm{~nm}$ wavelengths. In the case of dissolved or suspended components in the water the system estimates their concentration and, from data in EDB on the hydrodynamic parameters of the water body, it calculates their spatial distribution using the methods that process two-dimensional images.

\section{Data Processing Algorithms}

Spectroellipsometric measurements deliver spectrums that are considered as spectral images of water solutions. Space of spectral images is formed during the learning procedure realized in laboratory conditions when spectral images and chemical analysis are performed at the same time. Identification procedure to recognize spectral image of water solution is carried out with the use of the EDB where spectrums and their derivatives are storage during learning procedure. Standard of the EDB item is given in Table 2.

An identification of spectral image of unknown water solution is realized by means of comparison his vector identifier with elements of the EDB. Depending from used optical device spectral image of water solution can be represented by one or two vector-identifiers calculated with the use of rule described in Table 2. Final identification is realized by means of search in the EDB of vector identifiers which are minimal distance from considered vector-identifier $Q=\left\{X_{1}, \ldots, X_{n}\right\}$ of given water solution. Distance between vector-identifiers is calculated with the use of the following formula:

$$
\delta=\min _{n} \rho\left(Q-Q_{n}\right)=\frac{1}{2 n} \min _{i}\left[\sum_{j=1}^{n}\left|X_{j}-A_{j}^{i}\right|+\sqrt{\sum_{j=1}^{n}\left(X_{j}-A_{j}^{i}\right)^{2}}\right]
$$

Use of (1) gives better result in comparison with the application of other known criteria of closeness between spectral curves. That is why in this case there is minimal risk to miss the situation with dangerous pollution of water reservoir. In common case, usually the following methods are used:

- Cluster analysis. In this case two types of clusters are formed for $\operatorname{Cos} \Delta$ and $\operatorname{Tan} \Psi$ where $\Delta$ and $\Psi$ are ellipsometric angles corresponding to complex amplitude reflection coefficients for two different polarizations. Decision is made by weighted values (1) or independently for each polarization.

- Algorithm of discrepancy between spectra. It is assessed average distance between the ordinates for both spectra and spectrum of studied case and decision is made taking into account minimal value of this distance.

- Algorithm of discrepancy between etalon vectors. In this case, decision is made taking into consideration of minimal $\delta$.

- Inverse task solution. This algorithm is based on linear dependence of optical spectrum on the concentration of chemical elements in water solution. In this case, sub-definite system of linear algebraic equations is solved.

Last algorithm allows the obtainment of concentrations for chemical elements $\left\{y_{s}, s=1, \ldots, k\right)$ in aquatic environment. Its meaning is in the following procedure. As a result of the MCS functioning, at moment $t_{i}$ at the output of each channel $\lambda_{j}(j=1, \ldots, n)$ values $Z_{i j p}(i=1, \ldots, m ; p=1,2)$ are fixed, so that $Z_{i j 1}=\operatorname{Cos} \Delta_{j}+\xi_{i j}$ and $Z_{i j 2}=\operatorname{Tan}_{j}+\zeta_{i j}$, where $\Delta_{j}$ and $\Psi_{j}$ spectroellipsometric polarization angles, and $\xi_{i j}$ and $\zeta_{i j}$ are a random values (noises) with zero mean and dispersion $\sigma_{j}^{2}$. It is supposed that concentration of chemical elements linearly forms each spectrum:

$$
\sum_{s=1}^{k} a_{j s} y_{s}=Z_{j}^{1} \text { and } \sum_{s=1}^{k} b_{j s} y_{s}=Z_{j}^{2},
$$

where $Z_{j}^{p}=\frac{1}{M} \sum_{i=1}^{M} Z_{i j p}(p=1,2), M$ is number of spectrums averaged, empirical coefficients $a$ and $b$ are determined when studied aquatic environment has known parameters $\left\{y_{s}\right.$, $s=1, \ldots, k)$.

Table 2. Structure of standard spectral image of water solution. Notation: $A_{1}$ is the square occupied by spectral curve, $A_{2}$ is the maximal value of spectral curve, $A_{3}$ is the minimal value of spectral curve, $A_{4}$ is the distance between wavelengths with minimal and maximal values of spectral curve, respectively; $A_{5}$ is the maximal derivative of spectral curve; $A_{6}$ is the maximal value of second derivative of spectral curve; $A_{7}$ is the number of spectral curve maximums; $A_{8}$

\begin{tabular}{|c|c|c|c|c|c|c|c|c|c|c|}
\hline $\begin{array}{l}\text { Etalon } \\
\text { number }\end{array}$ & $\mathrm{A}_{1}$ & $\mathrm{~A}_{2}$ & $\mathrm{~A}_{3}$ & $\mathrm{~A}_{4}$ & $\mathrm{~A}_{5}$ & $\mathrm{~A}_{6}$ & $\mathrm{~A}_{7}$ & $\mathrm{~A}_{8}$ & $\mathrm{~A}_{9}$ & B \\
\hline 1 & $\mathrm{~A}_{11}$ & $\mathrm{~A}_{21}$ & $\mathrm{~A}_{31}$ & $\mathrm{~A}_{41}$ & $\mathrm{~A}_{51}$ & $\mathrm{~A}_{61}$ & $\mathrm{~A}_{71}$ & $\mathrm{~A}_{81}$ & $\mathrm{~A}_{91}$ & $\mathrm{~B}_{1}$ \\
\hline \multicolumn{11}{|l|}{$\ldots \ldots$} \\
\hline $\mathrm{n}$ & $\mathrm{A}_{1 \mathrm{n}}$ & $\mathrm{A}_{2 \mathrm{n}}$ & $\mathrm{A}_{3 \mathrm{n}}$ & $\mathrm{A}_{4 \mathrm{n}}$ & $\mathrm{A}_{5 \mathrm{n}}$ & $\mathrm{A}_{6 \mathrm{n}}$ & $\mathrm{A}_{7 \mathrm{n}}$ & $\mathrm{A}_{8 \mathrm{n}}$ & $\mathrm{A}_{9 \mathrm{n}}$ & $\mathrm{B}_{\mathrm{n}}$ \\
\hline
\end{tabular}
is the average value of spectral curve; $A_{9}$ is the wavelength correspond to average value of spectral curve; $B$ is the chemical element concentration. 
Practically $s<n$. In this case equations (2) are undetermined and their solution can be realized with the use of many methods. In [4] proposed analytical method based on solution dispersion minimization. This method is realized in the STW item.

\section{Empirical Results}

The SESAED was used in different laboratory and in-situ conditions. Table 3 shows example of vector-identifiers calculated for $\mathrm{CuSO}_{4} \quad 10 \%$ solution with use of Table 2 procedure. Table 4 gives experimental results which give possibility to compare above mention algorithms. Dependence of risk assessment as function of solution concentration is represented in Fig. 2. As it follows from these results risk to have high error under the solution identification is reduced when algorithm of Table 2 is used. We see that risk to have high error is growth with increase of chemical element concentration. It is caused that discrepancy between spectra is decreased with increase of chemicals concentration. In this case it is necessary to extend the database of spectral etalons.
The SESAED was used for in-situ experiments in South Vietnam [1,6]. Fig. 3 represents normalized spectral characteristics of studied water objects. It is seen that all spectral curves $\mathrm{I} / \mathrm{I}_{0}$ related to water quality intersect in the point $(510,0.3)$. This means that channel of $510 \mathrm{~nm}$ is invariant for studied reservoirs. As well it is followed that channels 570-690 are the most informative. Spectral images of pollution slicks for salt and oil on water surface are distinguished what gives possibility to recognize them with high reliability.

The SESAED was used for water quality control in Nuok Ngot Lagoon near Hung Lac (Vietnam) - 14 $9^{\prime} 0^{\prime \prime} \mathrm{N}$; $109^{\circ} 10^{\prime} 59.99^{\prime \prime} \mathrm{E}$, Saigon River in Hochiminh City area, and fish-farm reservoirs in $\mathrm{Ba} \mathrm{Ria}$ Province. Lagoon area was divided by ten sections location of which was uniformly distributed from the north to south. One measurement by means of the SESAED was performed in each of these locations and water sample was taken at the same time. Location 1 had coordinates $14^{\circ} 10^{\prime} \mathrm{N}, 109^{\circ} 10^{\prime} \mathrm{E}$. Location 10 corresponded to the lagoon mouth. Table 5 allows assessment of the SESAED precision. It is supposed that water samples were assessed with very high precision.

Table 3. An example of the SESAEC database element.

\begin{tabular}{|c|c|c|c|c|c|c|c|c|c|c|}
\hline Solution & $Q$ & \multicolumn{9}{|c|}{ Vector-identifiers } \\
\hline $\begin{array}{c}\mathrm{CuSO}_{4} \\
(10 \%)\end{array}$ & $Q_{\Delta}$ & 21.6 & 0.17 & 0.1 & 143 & 0.67 & 0.12 & 2 & 0.16 & 0.21 \\
\cline { 2 - 25 } & $Q_{\Psi}$ & 43.1 & 0.89 & 0.04 & 201 & 0.59 & 0.09 & 3 & 0.65 & 0.01 \\
\hline
\end{tabular}

Table 4. Comparatively assessment of algorithms for recognition of spectral images of water solutions.

\begin{tabular}{|c|c|c|c|c|}
\hline \multirow{2}{*}{ Object for study } & \multicolumn{4}{|c|}{ Identification algorithm and its error (\%) } \\
\cline { 2 - 5 } & Cluster analysis & Discrepancy between spectra & $\begin{array}{c}\text { Discrepancy between vector-etalons } \\
\text { with the use equation (1) }\end{array}$ & $\begin{array}{c}\text { Inverse task } \\
\text { solution }\end{array}$ \\
\hline $\mathrm{CuSO}_{4}$ & 15 & 12 & 8 & 7 \\
$\mathrm{NaCl}^{\mathrm{NaHCO}}$ & 17 & 11 & 5 & 5 \\
$\mathrm{NH}_{4} \mathrm{OH}$ & 16 & 10 & 5 & 5 \\
$\mathrm{ZnSO}_{4}$ & 21 & 13 & 8 & 6 \\
$\mathrm{Potassium}$ iodite & 22 & 12 & 6 & 6 \\
$\mathrm{Na}+\mathrm{Cu}+\mathrm{Zn}+\mathrm{Mn}+$ glucose & 13 & 10 & 9 & 8 \\
Furaciline & 18 & 9 & 5 & 5 \\
Bifidumbacterium & 23 & 11 & 4 & 4 \\
\hline
\end{tabular}

Table 5. Comparison of data received by means of the SESAED and in situ measurements in Nuok Ngot Lagoon.

\begin{tabular}{|c|c|c|c|c|c|c|c|c|}
\hline \multirow{2}{*}{ Location } & \multicolumn{2}{|c|}{ Water salinity $(\%)$} & \multicolumn{2}{|c|}{ Turbidity $(\mathrm{mg} / \mathrm{l})$} & \multicolumn{2}{|c|}{$\mathrm{pH}$} & \multicolumn{2}{c|}{$\mathrm{PO}_{4}^{-3}(\mathrm{mg} / \mathrm{l})$} \\
\hline \multirow{3}{*}{1} & SESAED & On-site & SESAED & On-site & SESAED & On-site & SESAED & On-site \\
\cline { 2 - 9 } 2 & 30.15 & 33.5 & 12.40 & 10 & 7.04 & 7.82 & 0.033 & 0.03 \\
3 & 23.14 & 26.0 & 24.78 & 21 & 7.71 & 7.79 & 0.034 & 0.03 \\
4 & 26.48 & 29.1 & 29.70 & 27 & 7.66 & 7.58 & 0.068 & 0.45 \\
5 & 27.37 & 32.2 & 26.88 & 24 & 8.01 & 7.78 & 0.023 & 0.02 \\
6 & 28.53 & 31.7 & 45.59 & 47 & 7.29 & 7.84 & 0.042 & 0.04 \\
7 & 28.40 & 26.3 & 29.97 & 27 & 7.22 & 7.52 & 0.082 & 0.10 \\
8 & 27.50 & 25.7 & 23.94 & 21 & 7.45 & 7.30 & 0.067 & 0.35 \\
9 & 26.16 & 25.4 & 34.58 & 38 & 7.21 & 7.75 & 0.023 & 0.02 \\
10 & 26.54 & 30.5 & 48.45 & 51 & 7.09 & 7.71 & 0.023 & 0.02 \\
\hline Average & 20.95 & 26.5 & 60.48 & 63 & 7.63 & 7.00 & 0.039 & 0.05 \\
error, \% & 10.7 & & 11.0 & & 6.0 & & 27.4 & \\
\hline
\end{tabular}




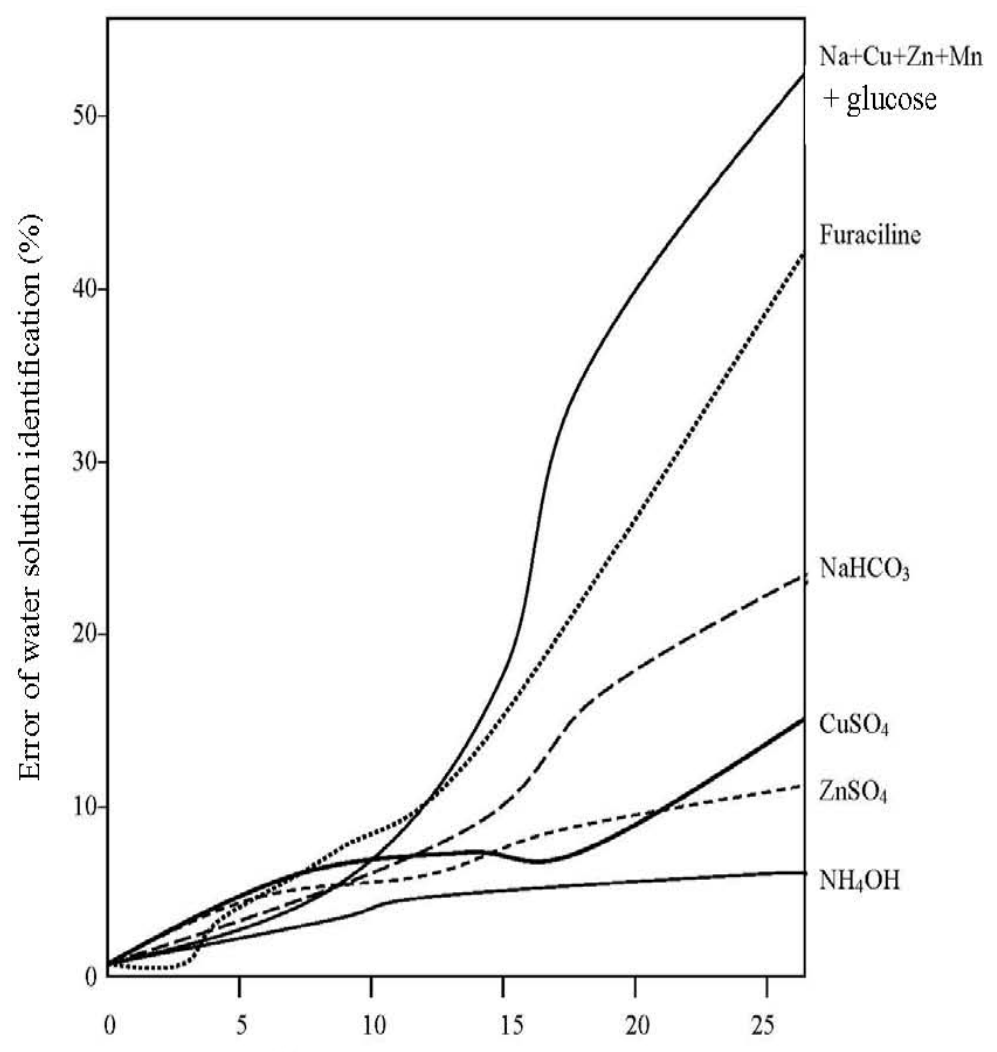

Water solution concentration $(\%)$

Figure 2. Dependence of spectral image identification on the solution concentration.

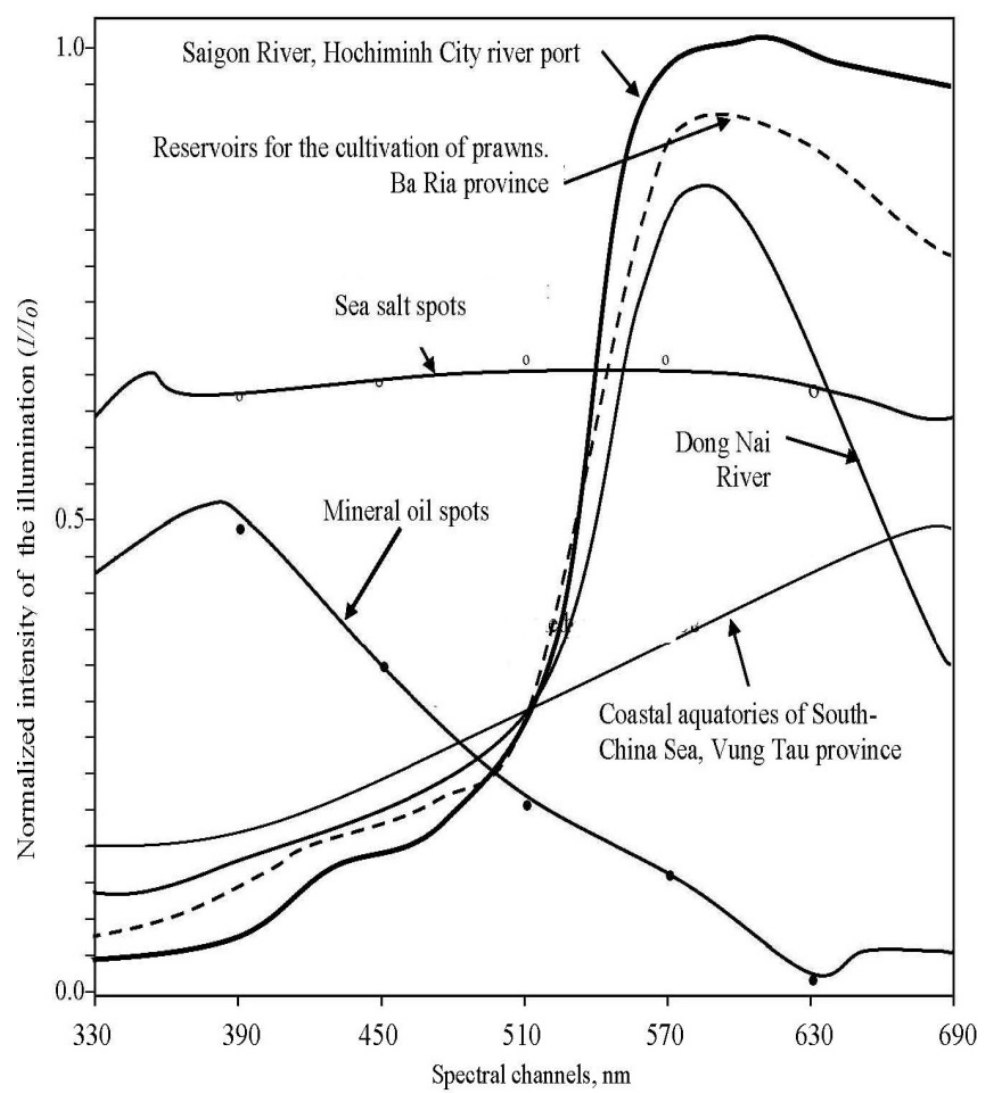

Figure 3. Spectral characteristics of some reservoirs located in South Vietnam. Notation: $I$ is registered illumination, $I_{0}$ is light intensity of free air 


\section{Conclusion}

The main objective of work is to create in future the compact information systems for monitoring the quality of aquatic environment and to investigate their potential efficiency. These systems are based on the combined application of spectroellipsometry methods and algorithms of training, classification, and identification. The realization of this objective will require the combined use of engineering and algorithmic tools providing real - time measurements and data processing. The technology of combined use of spectroellipsometry and algorithms of detection and classification will allow the creation of an original system of instrumental, algorithmic, modular and software tools for the collection and processing of data on the aquatic environment with forecasting and decision-making functions. The theoretical part of the work will include the use of methods of polarization optics, mathematical statistics, the theory of pattern recognition and mathematical modeling.

\section{Acknowledgements}

The reported study was partially supported by RFBR, research project No.13-07-00146.

\section{REFERENCES}

[1] Cao Van Phuong, Nguyen Boi Khue, Dang Manh Cuong, Nguyen Ngoc Tan, V.F. Krapivin, F.A. Mkrtchyan, and V.Yu. Soldatov. Synthesis of geoecological information-modeling systems. Proceedings of the Popov A.S. Russian Society for Radiotechnics, Electronics, and Communication. Serie: "Engineering Ecology",Vol. 5. 18-22.

[2] V.F. Krapivin , F.A. Mkrtchyan . Applications in study of environment. Proc. of the 8th Int. Conf. on Control Systems and Computer Science, 22-25 May 1991, Bucharest. Polytechnical Institute, Bucharest, 49-56.

[3] V.F. Krapivin, F.A. Mkrtchyan . Expert system for the operative environmental diagnostics. Proc. of the 21 st Int. Symposium on Okhotsk Sea \& Sea Ice, Mombetsu, Hokkaido, 19-24 February 2006, 128-131.

[4] F.A. Mkrtchyan, V.F. Krapivin, V.I. Kovalev, V.V. Klimov, A.I. Rukovishnikov, and S.P. Golovachev. An adaptive spectroellipsometric technology for the ecological monitoring of the aquatic environment. Proceeding of 25-th ACRS, Chiang-Mai, Thailand,2004, 13-15.

[5] Nguyen Xuan Man, V.F. Krapivin, F.A. Mkrtchyan , V.V. Klimov, V.I. Kovalev, A.I. Rukovishnikov, and V.Yu. Soldatov. Measurements and identification of the water solutions basing on the spectroellipsometer. Problems of Environment and Natural Resources, No.2, 2011, 3-22 [in Russian].

[6] P.I. Perov, V.I. Kovalev, A.I. Rukovishnikov, N.M. Rossukanyi, and W.H. Johnson . Hydrogen-sensitive palladium film study with precise and fast ellipsometers. Int. J.Electronics, Vol.76, No.5, 797-803 